\title{
Foresterie à base communautaire pour l'atténuation et l'adaptation dans la zone du Parc National des Volcans (PNV) au Rwanda (COBAM-ARECO)
}

\author{
Mukakamari Dancilla, Louis Bernard Cheteu
}

\section{Contexte}

La République du Rwanda est un pays de 26338 km² qui héberge une population d'environ 10 millions d'habitants, soit une densité physique de 416 habitants $/ \mathrm{km}^{2}$ (NISR, 2012). C'est le pays le plus densément peuplé d'Afrique continentale où la croissance démographique exerce une pression énorme sur les ressources naturelles, en particulier, sur les terres arables et les forêts. Sur une période de 41 ans, les superficies des forêts naturelles au Rwanda sont passées de 634000 ha à 221200 ha, soit une régression de 65,11 \% entre 1960 et 2002 (MINITERE, 2005). Les ressources forestières jouent un rôle important dans la protection de l'environnement, dans l'économie du pays ainsi que dans la vie quotidienne des populations. Plus de $97 \%$ de la population utilisent le bois comme source d'énergie, notamment pour la cuisson des aliments et l'éclairage (MINITERE, 2002).

Les préoccupations environnementales dans la région de Grands Lacs remontent à l'époque coloniale. Elles sont matérialisées par la création en 1925 du Parc Albert sur le côté congolais de la chaîne de montagnes, étendue en 1929 à la face rwandaise. Cette dernière partie est devenue le Parc National des Volcans après l'indépendance du Congo belge en 1960. Malgré le génocide et les massacres de 1994 qui ont paralysé les initiatives déjà entreprises, différentes mesures ont été adoptées grâce à la relance opérée par le Gouvernement d'Union Nationale, parmi lesquelles la ratification de la Convention sur la Diversité Biologique (CDB) en 1995, la Convention Cadre des Nations Unies sur les Changements Climatiques (CCNUCC) en1998.

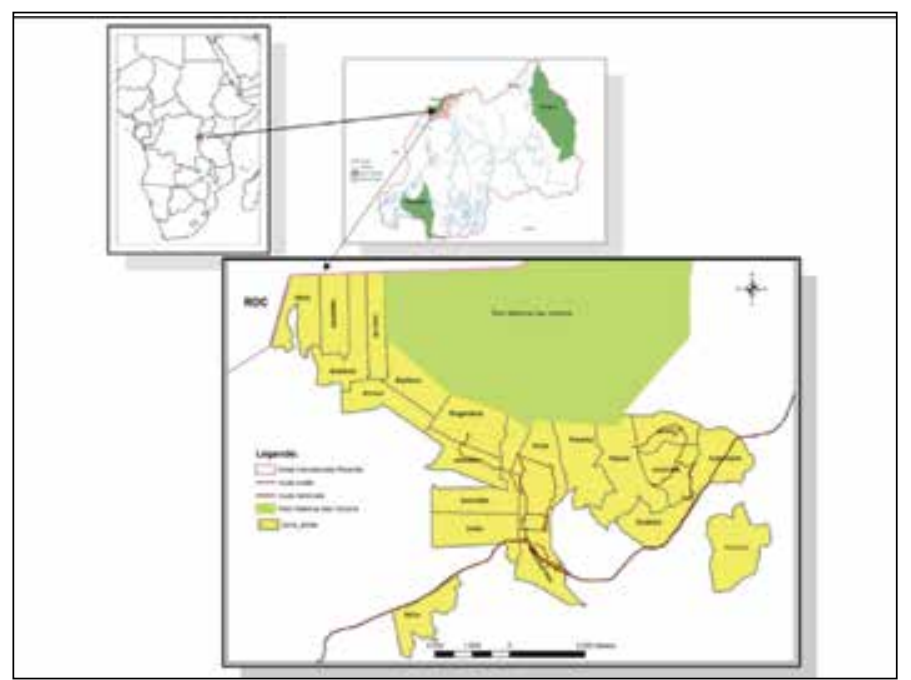

Figure 1. Carte de la zone du projet autour du Parc National des Volcans
Dans le cadre de la mise en œuvre de la CCNUCC, le Rwanda a élaboré sa première communication nationale sur les changements climatiques en 2005 et la deuxième en 2010. Le Programme d'Action National aux fins d'adaptation aux changements climatiques (PANA), élaboré et adopté en 2006, a ouvert les voies à une intégration des mesures et stratégies d'adaptation aux changements climatiques dans la Stratégie de développement économique et de réduction de la pauvreté (SDERP). La forte dégradation des terres cultivables due à l'érosion ou aux inondations, la tendance à la désertification des régions de l'Est et du Sud-Est, la baisse du niveau des lacs et cours d'eau due au déficit pluviométrique et aux sécheresses prolongées, la dégradation des forêts, sont autant d'effets néfastes d'origine climatique retenus. Le PANA présente par ailleurs des options d'adaptation et des propositions de projets prioritaires visant à accroître la capacité d'adaptation des populations et des secteurs vulnérables aux changements climatiques et contribuer au renforcement de la résilience des écosystèmes fortement fragilisés. Parmi ces derniers, des projets de conservation et de protection des terres contre l'érosion et les inondations et le développement des sources d'énergie alternatives au bois se situent à l'interface de l'adaptation et de l'atténuation.

Le projet « Foresterie à base communautaire pour l'atténuation et l'adaptation dans la zone du Parc National des Volcans (PNV) au Rwanda » s'inscrit dans cette dynamique. II fait partie d'une série de cinq projets pilotes initiés avec l'appui technique du

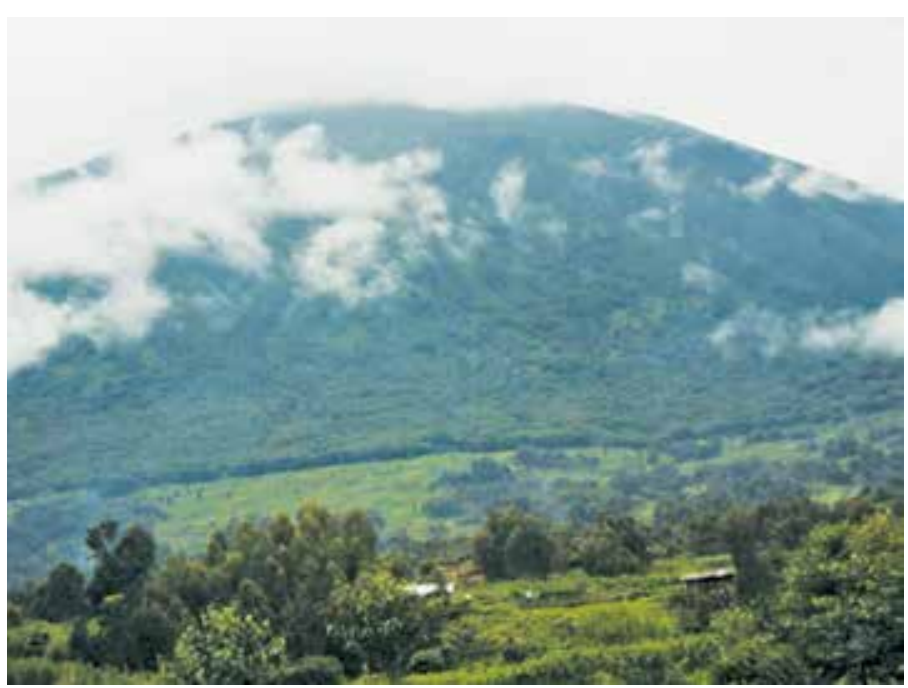

Figure 2. Un aperçu du Parc National des Volcans 




Figure 3. Paysage dénudé par l'agriculture intensive

projet Changement climatique et forêts dans le Bassin du Congo : synergies entre adaptation et atténuation, COBAM en abrégé. C'est un projet initié par le Centre de recherche forestière internationale (CIFOR), dans le cadre de l'aide accordée par la Banque africaine de développement (BAD) à la Communauté économique des États de I'Afrique centrale (CEEAC), pour financer le Programme d'appui à la conservation des écosystèmes du Bassin du Congo (PACEBCo).

\section{Le Site}

La zone du projet est située au Nord-Ouest du Rwanda, dans le versant Sud du massif des Virunga et s'étend sur une superficie d'environ 60,4 km2. Elle couvre les Districts de Nyabihu et Rubavu de la Province de l'Ouest et comprend cinq Secteurs administratifs (Jenda, Mukamira, Bigogwe, Kabatwa et Bugeshi) et 5 Cellules.

L'agriculture constitue la principale activité économique pour plus de $90 \%$ de la population (ARECO-RWANDA NZIZA, 2011) avec comme principales cultures : la pomme de terre, le maïs, le prunier du Japon, le sorgho, le haricot, le petit pois, les colocases et le blé. La taille moyenne de l'exploitation est évaluée de 0,25 à 0,8 ha, car la rareté des terres est un facteur limitant dans cette zone où la densité de la population atteint 650 habitants $/ \mathrm{km}^{2}$. Les autres activités observées sont : l'élevage, le petit commerce, l'artisanat, l'exploitation des bambous, le reboisement et l'écotourisme.

\section{Vulnérabilité des populations riveraines aux changements climatiques}

L'évaluation de la vulnérabilité des systèmes naturels et humains faite par Pavageau et al. (2013) dans le site du projet a montré que les groupes communautaires, les ressources naturelles et les activités sont touchés différemment par diverses perturbations liées au climat. Les personnes vulnérables sont surtout les veuves,

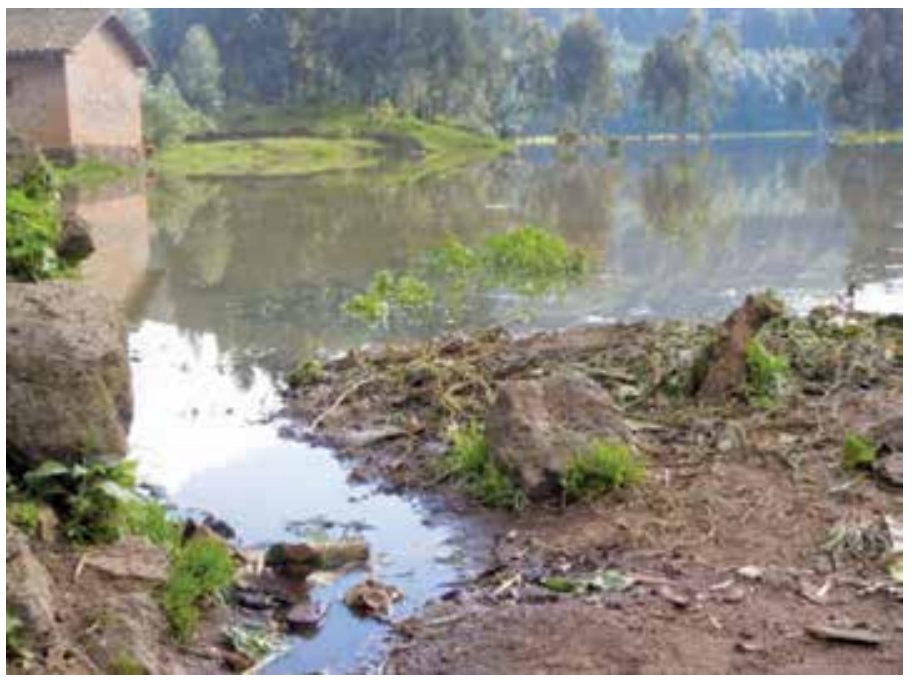

Figure 4. Inondation des bas-fonds après des pluies diluviennes

les orphelins, les vieillards, les personnes handicapées, les enfants responsables de familles, et les personnes historiquement marginalisées dites « Abahejwe inyuma n'amateka ».

Avec une densité de population élevée, les conflits fonciers sont latents. L'agriculture, qui est le principal moyen de subsistance de la majorité de la population, est l'activité la plus touchée par les perturbations liées au climat. La non-prise en compte des conflits fonciers et des inégalités existantes dans le processus récent de formalisation des titres fonciers constitue un obstacle majeur à l'élaboration de stratégies d'adaptation pour les groupes défavorisés qui n'ont pas accès aux terres.

Les villages situés autour du PNV sont confrontés à une série de risques climatiques ayant de graves impacts. Les pluies torrentielles qui provoquent des inondations et glissement de terrain, le prolongement des saisons pluvieuses, les vents violents et les poches de sécheresse constituent de véritables fardeaux sur les activités de survie des populations qui appliquent déjà de mauvaises pratiques culturales, facteurs importants de l'érosion des terres. Les effets de la perturbation du climat sont nombreux: diminution considérable des rendements agricoles, famine, accentuation des maladies comme la fièvre typhoïde, apparition des nouvelles maladies, augmentation des sans-abris à cause de l'écroulement des maisons et de la pollution des eaux.

Des outils d'analyse participative des perceptions utilisés en 2012 avec des groupes focaux ont permis de faire ressortir l'acuité des impacts positifs ou négatifs des aléas climatiques sur les activités ou la disponibilité des produits. Les résultats sont consignés dans le tableau 1:

Tableau 1. Degré d'impact des aléas climatiques sur les activités de survie dans la zone du projet



Légende : $0=$ aucun impact ; $5=$ impact très élevé $;+=$ positif ; - = négatif. 


\section{Causes et agents de la déforestation et de la dégradation des forêts}

Le taux de déforestation, qui se situerait à 4,14\% entre 2000-2005, est incontestablement le plus élevé de la région à cause de la très haute densité des populations qui peut atteindre 650 habitants au $\mathrm{km}^{2}$ par endroits (Bodson et al., 2009). Les groupes communautaires exercent des pressions de tous ordres sur les ressources naturelles entraînant la modification des écosystèmes et la perte de la biodiversité. L'agriculture de rente à l'instar de la culture de pyrèthre, l'exploitation illégale des minerais, l'exploitation anarchique des forêts notamment pour satisfaire les besoins en bois d'œuvre et énergie, le prélèvement massif des bambous pour la construction de l'habitat, l'agriculture, la confection de différents objets à usage domestique, constituent une réelle menace pour les forêts, particulièrement dans le PNV. Les feux sont relativement rares, mais leurs effets sont dévastateurs.

\section{Comment le projet compte- $\mathrm{t}$ - il contribuer à trouver des solutions à ces problèmes ?}

Le projet vise à contribuer à la réduction de la déforestation, de la dégradation du PNV et à l'adaptation des communautés riveraines à la variabilité et au changement climatique.

\section{Objectifs du projet}

Ce projet vise les objectifs suivants:

- Conscientiser et renforcer les capacités des acteurs en matières d'atténuation et d'adaptation face à la variabilité climatique et à la vulnérabilité liée au changement climatique

- Conserver le couvert forestier et par conséquent les réserves de carbone, la biodiversité et la capacité des forêts à fournir des biens et services;

- Protéger les bassins versants et prévenir l'érosion du sol ;
- Renforcer les capacités des populations locales pour une gestion productive et durable des ressources naturelles et la création des activités génératrices de revenus ;

- Mettre en place un mécanisme de suivi et de coordination du projet en vue de garantir le succès et la durabilité des actions.

Activités

Pour atteindre les objectifs qui lui sont assignés, les activités du projet, identifiées de façon participative, sont classées en quatre catégories distinctes:

- $\quad$ Protection du PNV : Conscientisation et formation des communautés à la surveillance, au suivi et à la protection du parc national;

- Reboisement, intensification agricole et aménagement des terrasses progressives pour lutter contre l'érosion;

- Promotion de l'agroforesterie et intensification agricole ;

- Économie du bois-énergie par la production et dissémination des foyers améliorés en faveur des communautés ;

- Promotion des activités génératrices de revenus tels que l'élevage des petits ruminants, la formation des jeunes à la confection des vêtements, le développement du tourisme écologique et culturel et l'apiculture moderne.

\section{Groupes cibles}

Le projet cible les communautés vivant autour du PNV, particulièrement, les femmes, les ex-braconniers, les artisans et les populations historiquement marginalisées. Les ex-braconniers sont en majorité des jeunes qui ont abandonné les activités de braconnage et de coupe du bois dans le parc. Les femmes et les populations autochtones sont particulièrement reconnues pour leur vulnérabilité mais aussi pour leur rôle potentiel dans la conservation des forêts. II est à noter que les cadres et les techniciens des institutions environnementales étatiques bénéficieront aussi directement du projet.

Tableau 2. Bénéfices attendus du projet

\begin{tabular}{|c|c|c|c|c|}
\hline \multirow{2}{*}{ Composantes du projet } & \multicolumn{4}{|c|}{ Bénéfices } \\
\hline & Atténuation & Adaptation & Biodiversité & Bien-être des communautés \\
\hline $\begin{array}{l}\text { Conscientisation et } \\
\text { renforcement des capacités } \\
\text { des acteurs en matière } \\
\text { d'atténuation et d'adaptation }\end{array}$ & $\begin{array}{l}\text { Capacités des } \\
\text { parties prenantes } \\
\text { renforcées } \\
\text { en matière } \\
\text { d'atténuation }\end{array}$ & $\begin{array}{l}\text { Capacités des parties prenantes } \\
\text { renforcées en matière } \\
\text { d'adaptation }\end{array}$ & $\begin{array}{l}\text { Meilleure connaissance } \\
\text { des concepts d'atténuation } \\
\text { et d'adaptation au } \\
\text { changement climatique et } \\
\text { de ses conséquences sur la } \\
\text { biodiversité }\end{array}$ & $\begin{array}{l}\text { Amélioration des connaissances des } \\
\text { parties prenantes sur les causes et } \\
\text { les effets du changement climatique }\end{array}$ \\
\hline $\begin{array}{l}\text { Conservation du couvert } \\
\text { forestier, restauration des } \\
\text { paysages et prévention de } \\
\text { l'érosion des sols }\end{array}$ & $\begin{array}{l}\text { Augmentation du } \\
\text { stock de carbone. }\end{array}$ & $\begin{array}{l}\text { Résilience aux aléas climatiques } \\
\text { à travers la protection des sols, } \\
\text { et augmentation de la capacité } \\
\text { adaptative grâce aux sources des } \\
\text { revenus. }\end{array}$ & $\begin{array}{l}\text { Protection de la biodiversité } \\
\text { due à l'amélioration de la } \\
\text { résilience des écosystèmes. }\end{array}$ & $\begin{array}{l}\text { Nouvelle source de revenus pour } \\
\text { les communautés et de nombreux } \\
\text { services écosystémiques sécurisés. }\end{array}$ \\
\hline $\begin{array}{l}\text { Renforcement des capacités } \\
\text { des populations locales } \\
\text { pour une gestion productive } \\
\text { et durable des ressources } \\
\text { naturelles }\end{array}$ & $\begin{array}{l}\text { Augmentation du } \\
\text { stock de carbone à } \\
\text { travers la réduction } \\
\text { de la déforestation } \\
\text { liée à la recherche } \\
\text { de bois énergie }\end{array}$ & $\begin{array}{l}\text { Meilleure protection contre les } \\
\text { inondations et les glissements } \\
\text { des terres. }\end{array}$ & $\begin{array}{l}\text { Protection de la biodiversité } \\
\text { par le développement des } \\
\text { activités alternatives à la } \\
\text { chasse et l'amélioration de la } \\
\text { résilience des écosystèmes. }\end{array}$ & $\begin{array}{l}\text { Des maisons et autres structures } \\
\text { plus protégées, et un meilleur } \\
\text { état nutritionnel grâce aux foyers } \\
\text { améliorés. }\end{array}$ \\
\hline $\begin{array}{l}\text { Création des activités } \\
\text { génératrices de revenus pour } \\
\text { les communautés }\end{array}$ & $\begin{array}{l}\text { Augmentation } \\
\text { du stock de } \\
\text { carbone à travers } \\
\text { l'agroforesterie. }\end{array}$ & $\begin{array}{l}\text { Contribution à l'accroissement } \\
\text { des actifs financiers, sociaux et } \\
\text { socioéconomiques renforçant } \\
\text { la capacité adaptative }\end{array}$ & Conservation de la biodiversité & $\begin{array}{l}\text { Nouvelles sources de revenus pour } \\
\text { les communautés et amélioration } \\
\text { de l'état nutritionnel à travers des } \\
\text { fruits et produits forestiers non } \\
\text { ligneux (miel). }\end{array}$ \\
\hline $\begin{array}{l}\text { Mise en place d'un } \\
\text { mécanisme de suivi et de } \\
\text { coordination du projet en } \\
\text { vue de garantir le succès et la } \\
\text { durabilité des actions }\end{array}$ & $\begin{array}{l}\text { Suivi-évaluation } \\
\text { des activités liées à } \\
\text { l'atténuation }\end{array}$ & $\begin{array}{l}\text { Evaluation continue des activités } \\
\text { de réduction de la vulnérabilité } \\
\text { des communautés locales. }\end{array}$ & $\begin{array}{l}\text { Meilleure connaissance de } \\
\text { l'impact du changement } \\
\text { climatique sur la biodiversité }\end{array}$ & $\begin{array}{l}\text { Suivi du changement des attitudes } \\
\text { et comportements des acteurs. } \\
\text { Renforcement des capacités des } \\
\text { communautés sur le suivi-évaluation } \\
\text { participatif. }\end{array}$ \\
\hline
\end{tabular}


Bénéfices attendus de la mise en œuvre du projet

La mise en œuvre du projet va permettre de réduire la déforestation et la dégradation des ressources naturelles d'une part, d'augmenter la séquestration du carbone à travers l'agroforesterie d'autre part. Par ailleurs, les activités d'adaptation aux changements climatiques et génératrices de revenus prévues dans le projet vont assurer à la population des gains nécessaires à l'amélioration des conditions de vie et du renforcement des capacités d'adaptation.

Il est attendu du projet des bénéfices divers notamment dans les domaines de l'adaptation, de l'atténuation et de la gestion de la biodiversité, avec des impacts certains sur le bien-être des populations, tels que le montre le tableau 2.

\section{Les organisations de mise en œuvre du projet}

Le projet est mis en œuvre par ARECO-RWANDA NZIZA qui est une organisation d'environnement et de développement créée en 1992. Elle contribue à la mise en œuvre des politiques nationales à travers la mobilisation et l'encadrement des communautés pour la gestion durable des ressources naturelles. ARECO opère dans les zones des aires protégées dont le Parc National des Volcans. Elle apporte un soutien technique aux communautés locales, particulièrement les femmes, les jeunes, les ex-braconniers, les populations historiquement marginalisées, dans des initiatives de gestion participatives des ressources naturelles dont la foresterie.

Le CIFOR à travers le COBAM apporte un appui technique à la planification et la mise en œuvre des activités du projet et assure le suivi-évaluation de la mise en œuvre des activités du projet.

\section{Contacts}

Pour toute information complémentaire, veuillez contacter:

- Madame Dancilla Mukakamari; Coordinatrice de I'Association Rwandaise des Ecologistes (ARECO-RWANDA NZIZA); PO Box 3967 Kigali-Rwanda; Opposite Kigali International Airport; Tel: + (250) 0788521732;

Courriel: dmukakamari@arecorwandanziza.org, info@arecorwandanziza.org

Site internet: www.arecorwandanziza.org

- Madame Anne Marie Tiani;

Coordinatrice du COBAM: a.tiani@cgiar.org
- CIFOR Bureau régional pour l'Afrique Centrale: cifor.cameroon@cgiar.org

- PACEBCo : info@pacebco-ceeac.org

\section{Référence bibliographique}

ARECO-RWANDA NZIZA. (2011). Note d'idée de projet (NIP) Appui à la promotion de la foresterie à base communautaire pour la conservation de la biodiversité, l'amélioration des conditions de vie et la lutte contre le changement climatique dans la zone du Parc National des Volcans (PNV) au Rwanda. 26 p.

Bodson T., Bubendwa R., Languy M. de Marcken P. (2009). Dans (eds.) de Wasseige, C., Devers, D., de Marcken, R., Eba'a Atyi, R., Nasi, R. et Mayaux, P. (2009). Les forêts du Bassin du Congo : Etat des forêts 2008. 375-384. Office des publications de l'Union européenne, Luxembourg.

Ministère des ressources naturelles (2012). Deuxième communication nationale initiale relative à la Convention cadre des nations unies sur les changements climatiques, mai 2012; Ministère des ressources naturelles Kigali. $217 \mathrm{p}$.

Ministère des terres, de l'environnement, des forêts, de l'eau et des mines (MINITERE), (2005). Communication nationale initiale relative à la Convention cadre des nations unies sur les changements climatiques, juin 2005; Ministère des terres, de l'environnement, des forêts, de l'eau et des mines. Kigali. $121 \mathrm{p}$.

Ministère des terres, de l'environnement, des forêts, de l'eau et des mines (MINITERE), (2006). Programme d'Action National pour l'Adaptation (PANA). Décembre 2006. Kigali, 85 p.

Ministère des terres, de la réinstallation et de la protection de l'environnement (MINITERE), (2002). Rapport National du Rwanda sur le développement durable et la mise œuvre de l'Agenda 21 ; Sommet mondial sur le développement durable ou Rio+10, Johannesburg, septembre 2002 ; Ministère des terres, de la réinstallation et de la protection de l'environnement. Kigali. 75 p.

National Institute of Statistics of Rwanda (NISR), (2012) Rwanda Fourth Population and Housing Census (RPHC4). Thematic Report: Population size, structure and distribution. January 2014. Kigali Ministry of Finance and Economic Planning (MINECOFIN), $102 \mathrm{p}$.

Pavageau C., Y. M. Bele et A.M. Tiani. (2012). Enjeux et potentialités pour des synergies entre l'adaptation et l'atténuation dans le bassin du Congo, poster

Pavageau, C., R. Butterfield et A. M. Tiani (2013). Current vulnerability in the Virunga landscape, Rwanda. Bogor, Indonésie, Centre de recherche forestière internationale (CIFOR) : $4 p$.

RUZIBIZA Marcel (2012). Ateliers sur les écosystèmes et la vulnérabilité climatique.18-24 mai 2012 à Muzanze-Rwanda. Rapport interne.

Promoteur du projet

L'Association Rwandaise des Ecologistes (ARECO-RWANDA NZIZA) ; PO Box 3967 Kigali-Rwanda ; Opposite Kigali International Airport Tel: + (250) 0788521732 ; Courriel : arecorwa@yahoo.fr

Site internet : www. arecorwandanziza.org

COBAM est mis en œuvre par le Centre pour la recherche forestière internationale (CIFOR), dans le cadre du support de la Banque africaine de développement (BAD) à la Communauté économique des Etats de l'Afrique centrale (CEEAC), pour financer le Programme d'appui à la conservation des écosystèmes du Bassin du Congo (PACEBCo).
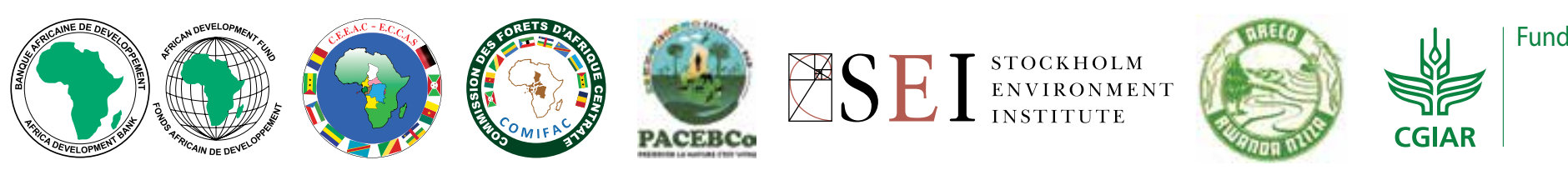

\section{cifor.org/cobam} recherche scientifique qui contribue à l'élaboration des politiques et des pratiques affectant les forêts dans les pays en développement. Le CIFOR est membre du Consortium du CGIAR. Son siège est situé à Bogor en Indonésie et il est 